\title{
Allelic Polymorphisms of Human Fc $\gamma$ Receptor IIA and Fc $\gamma$ Receptor IIIB Independent Mechanisms for Differences in Human Phagocyte Function
}

\author{
Jane E. Salmon, Jeffrey C. Edberg, Nina L. Brogle, and Robert P. Kimberly \\ Department of Medicine, The Hospital for Special Surgery and The New York Hospital, \\ The Cornell University Medical College, New York 10021
}

\begin{abstract}
Two different allelic polymorphisms among the isoforms of human Fc $\gamma$ receptors have been defined: the low-responder (LR)high-responder (HR) polymorphism of huFc $\gamma$ RIIA expressed on both PMN and monocytes and the NA1-NA2 polymorphism of the neutrophil FeyRIII (huFcrRIIIB). To address the issues of whether the LR-HR polymorphism has a significant impact on Fc $\gamma R$-mediated functions in human blood cells and whether any differences in LR-HR might be related to higher FcrR-mediated phagocytosis in NA1 donors, we examined FcrR-specific binding and internalization by donors homozygous for the two huFcrRIIA alleles. PMN from LR homozygotes showed consistently higher levels of internalization of erythrocytes opsonized with pooled human IgG (E-hIgG). The absence of an LR-HR phagocytic difference with erythrocytes opsonized with either anti-FcrRIIA MAb IV.3 or rabbit IgG, as opposed to E-hIgG, suggested that the Fc piece of the opsonin might be important for this LR-HR difference. Accordingly, we studied HR and LR homozygotes with human IgG subclass-specific probes. Both PMN (independent of huFc $\gamma$ RIIIB phenotype) and monocytes from $L R$ donors bound and internalized erythrocytes coated with human IgG2 (EhIgG2) efficiently, whereas phagocytes from HR donors did so poorly. E-hIgG2 internalization was completely abrogated by blockade of the ligand binding site of huFcrRIIA with IV.3 Fab, indicating that huFc $\gamma$ RIIA is essential for the handling of hIgG2 and that the mechanism of the LR-HR phagocytic difference is at the level of ligand binding to huFcrRIIA. In contrast, the difference in internalization of E-hIgG between NA1 and NA2 homozygous donors was independent of the huFcrRIIA phenotype and did not manifest differences in ligand binding. Thus, the two known allelic polymorphisms of human FcrR have distinct and independent mechanisms for altering receptor function, which may influence host defense and immune complex handling. (J. Clin. Invest. 1992. 89:1274-1281.) Key words: IgG subclasses • phagocytosis • monocyte $\bullet$ neutrophil
\end{abstract}

\section{Introduction}

Two different allelic polymorphisms among the isoforms of human $\mathrm{Fc} \gamma$ receptors have been defined. The NA1-NA2 poly-

Address reprint requests to Dr. Salmon, The Hospital for Special Surgery, 535 East 70th Street, New York, NY 10021.

Received for publication 14 June 1991 and in revised form 6 December 1991.

J. Clin. Invest.

(c) The American Society for Clinical Investigation, Inc.

$0021-9738 / 92 / 04 / 1274 / 08 \$ 2.00$

Volume 89, April 1992, 1274-1281 morphism of the neutrophil Fc $\gamma$ RIII (huFc $\gamma$ RIIIB) ${ }^{1}$ reflects several differences in amino acid sequence and two potential glycosylation sites (1-5). The low-responder (LR)-high-responder (HR) polymorphism of huFcyRIIA reflects two amino acid substitutions at positions 27 and 131, with an arginine residue at amino acid position 131 being critical for efficient binding of mIgG1 (6-9). For each of these allelic systems, there has been substantial interest in identifying their functional consequences and the molecular mechanisms underlying any differences. We have shown that individuals homozygous for the NA1 and NA2 alleles of huFc $\gamma$ RIIIB have distinct phagocytic capacities (10).

The recent observation by Warmerdam et al. (8) that position 131 of Fc $\gamma$ RIIA expressed in transfected fibroblasts influences the capacity for binding human IgG2 (hIgG2) suggests that this polymorphism may also be important in human systems. Using a large donor population with defined huFc $\gamma$ RIIA alleles, we examined the effect of this polymorphism on quantitative phagocytosis by human phagocytes. Our results revealed that homozygous $L R$ donors have a higher capacity to internalize erythrocytes (E) opsonized with human IgG (EhIgG) than homozygous HR donors. Further analysis of these homozygous donors indicated that, when studied with human IgG (hIgG) subclass-specific probes, PMN and monocytes from both $L R$ and HR subjects are able to bind and internalize E-IgG1 comparably. With E-IgG2, both cell types from homozygous LR subjects bind and internalize efficiently whereas cells from HR donors do so poorly. Thus, with intact human phagocytes, the LR-HR polymorphism influences both ligand binding to huFc $\gamma$ RIIA and subsequent $\mathrm{Fc} \gamma \mathrm{R}$-mediated phagocytosis.

This difference in Fc $\gamma \mathrm{R}$-mediated phagocytosis, coupled with recent data localizing the genes for both huFc $\gamma$ RII and huFc $\gamma$ RIII to human chromosome 1 (11-13), raised the possibility that an association between the NA alleles of huFc $\gamma$ RIIIB and the LR-HR alleles of huFc $\gamma$ RIIA might explain the difference in Fc $\gamma$ R-mediated phagocytosis between NA1 homozygous and NA2 homozygous donors. Thus, we also examined

1. Abbreviations used in this paper: AI, adherence index; ANOVA, analysis of variance; E, erythrocytes; EA, IgG-sensitized bovine erythrocytes; $E_{B}$, biotinylated erythrocytes; $E_{B A}$, streptavidin-coated $E_{B} ; E$ hIgG, E coated with pooled hIgG; E-hIgG1, E coated with human IgG1; E-hIgG2, E coated with human IgG2; E-hIgG3, E coated with human IgG3; E-hIgG4, E coated with human IgG4; E-IV.3, E coated with IV.3 $\mathrm{F}\left(\mathrm{ab}^{\prime}\right)_{2}$; hIgG, human IgG containing mixed subclasses; HR, high-responder allele of huFc $\gamma$ RIIA; huFc $\gamma R$, receptors for Fc portion of IgG in human cells; huFc $\gamma \mathrm{RIIA}, 40-\mathrm{kD}$ receptor on human neutrophils and monocytes for Fc portion of IgG; huFc $\gamma$ RIIIB, 50-78-kD receptor on human neutrophils for Fc portion of IgG; LR, low-responder allele of huFc $\gamma$ RIIA; MCF, mean channel log fluorescence units; mIgG1, murine IgG1 myeloma protein; PE, phycoerythrin; PI, phagocytic index. 
the differences in phagocytosis for NA1 and NA2 homozygotes matched for expression of the LR-HR polymorphism. Phagocytosis of $E$ coated with hIgG was higher for NA1 donors independent of the LR-HR phenotype. Furthermore, there were no differences in E-hIgG binding by cells expressing either NA1 or NA2 alleles. The mechanism of the phagocytic difference, therefore, appears to be distal to ligand binding. These data demonstrate that the two recognized allelic polymorphisms of human $\mathrm{Fc} \gamma$ receptors have independent mechanisms for mediating functionally apparent differences in Fc $\gamma \mathrm{R}$-mediated phagocytosis. These differences may have important implications for human disease.

\section{Methods}

Subjects. Peripheral blood was collected from 32 disease-free volunteers who ranged in age from 20 to 56 yr $(34 \pm 8$ yr [mean \pm SD] $)$. Protocols for these studies were approved by the Institutional Committee on Human Rights in Research.

Determination of NA antigens by leukoagglutination. Typing for neutrophil-specific antigens, NA1 and NA2, was performed by leukoagglutination as described by Lalezari $(2,14)$, with a panel of antiNA1 and anti-NA2 allosera kindly provided by Dr. David Stroncek of the American Red Cross Neutrophil Serology Reference Laboratory, St. Paul, MN. The assignment of NA type was confirmed by immunoprecipitation and flow cytometry with MAbs CLB-FcR gran 1, CLBgran 11 , and GRM1 $(15,16)$.

Determination of $L R-H R$ alleles by flow cytometry. Phenotyping of donors for the LR-HR alleles of huFc $\gamma$ RIIA was performed by quantitative flow cytometry using MAbs $41 . \mathrm{H} 16$ and IV.3 as described by Gosselin et al. (17). Phenotypic assignment was corroborated by antiCD3 mitogenesis assays as described below.

Reagents. HBSS, RPMI 1640, and FCS were from Gibco Laboratories, Grand Island, NY. FCS was heat inactivated at $56^{\circ} \mathrm{C}$ for $60 \mathrm{~min}$ before use. FITC-conjugated rabbit anti-human IgG $\mathrm{F}\left(\mathrm{ab}^{\prime}\right)_{2}$ FITCconjugated goat anti-rabbit IgG $\mathrm{F}\left(\mathrm{ab}^{\prime}\right)_{2}$, and phycoerythrin (PE)-conjugated goat anti-mouse IgG $\mathrm{F}\left(\mathrm{ab}^{\prime}\right)_{2}$ was purchased from Tago, Inc. Burlingame, CA. All conjugated reagents were absorbed against human mononuclear cells before use. Sulfosuccinimidobiotin (SulfoNHS-biotin), sulfosuccinimidyl-6-(biotinamido) hexanoate (NHS-LCbiotin), and streptavidin were obtained from Pierce Chemical Co., Rockford, IL. FMLP (Sigma Chemical Co., St. Louis, MO) was dissolved in American Chemical Society grade DMSO for a stock concentration of $10^{-3} \mathrm{M}$ and stored at $-20^{\circ} \mathrm{C}$.

MAb 3G8 (mIgG1) recognizing huFc $\gamma$ RIII (CD16) was kindly provided by Dr. J. Unkeless, Mt. Sinai Medical Center, New York (18) The MAb IV.3 (mIgG2b) and IV.3 Fab fragments recognizing huFc $\gamma$ RII (CD32) and 3G8 $\mathrm{F}\left(\mathrm{ab}^{\prime}\right)_{2}$ were purchased from Medarex Inc., (West Lebanon, NH) (19). Silver stain analysis of the IV.3 Fab fragment preparation and the $3 \mathrm{G} 8 \mathrm{~F}\left(\mathrm{ab}^{\prime}\right)_{2}$ preparation indicated that there was no intact IgG. The MAb $41 \mathrm{H} .16$ (mIgG2a) recognizing huFc $\gamma$ RII was generously provided Dr. Thomas Zipf, University of Texas Cancer Center, Houston, TX (20). Leu4 and OKT3 (IgG1 and IgG2a anti-CD3, respectively) were provided by Dr. Robert Evans, Memorial Sloan-Kettering Cancer Center, New York. Isotype controls (mIgG1 [MOPC21] and mIgG2a [UPC10]) were purchased from Sigma Chemical Co.

Purified hIgG1 and hIgG2 myeloma proteins were obtained from Dr. Elliot Osserman of Columbia University, New York. hIgG3 and hIgG4 myeloma proteins were obtained from The Binding Site, Inc. (San Diego, CA). A second set of preparations of hIgG1, hIgG2, hIgG3, and hIgG4 were obtained from Calbiochemic-Behring Corp., San Diego, CA. Normal pooled hIgG was obtained from Sigma Chemical Co.

Preparation of cells. Fresh anticoagulated human peripheral blood was separated by centrifugation through a discontinuous two-step Ficoll-Hypaque gradient (10). PMNs were isolated from the lower inter- face and washed with HBSS. Contaminating E were lysed with hypotonic saline $(0.02 \% \mathrm{NaCl})$ for $20 \mathrm{~s}$ followed by $0.16 \% \mathrm{NaCl}$ and a final wash with HBSS. Mononuclear cells were isolated from the upper Ficoll-Hypaque interface and washed with HBSS. The percent monocytes within the mononuclear cell layer was determined by peroxidase staining. After final washes, all cells were resuspended to $5 \times 10^{6} \mathrm{PMNs}$ or monocytes $/ \mathrm{ml}$.

Preparation of $E$. Antibody-coated erythrocytes (EA) were prepared by incubating bovine $\mathrm{E}$ with rabbit $\mathrm{IgG}$ anti-bovine $\mathrm{E}$ antibody (Cappel Laboratories, Cochranville, PA) for $1 \mathrm{~h}$ at $37^{\circ} \mathrm{C}$. The cells were washed and resuspended at $10^{8}$ cells $/ \mathrm{ml}$ in RPMI and $20 \%$ FCS (21). The standard amount of antibody used was a fourfold dilution of the minimum agglutinating titer and resulted in maximum phagocytosis of EA by monocytes.

E were coupled to pooled or subclass-specific hIgG by a biotin-avidin technique (22). To prepare biotinylated $\mathrm{E}\left(\mathrm{E}_{\mathrm{B}}\right), 0.5 \mathrm{ml}$ of $\mathrm{E}\left(1 \times 10^{9}\right.$ cells $/ \mathrm{ml}$ ) in $0.1 \mathrm{M}$ carbonate buffer ( $\mathrm{pH} 8.6$ ) was incubated with sulfoNHS-biotin $(500 \mu \mathrm{g} / \mathrm{ml})$ for $20 \mathrm{~min}$ at $4^{\circ} \mathrm{C}$ with mixing, followed by three washes with buffer containing divalent cations as follows (mM): ( 2.5 veronal buffer, $\mathrm{pH} 7.4 ; 146 \mathrm{NaCl}, 0.05 \%$ gelatin, $0.15 \mathrm{CaCl}_{2}$, and $0.05 \mathrm{MgCl}_{2}$ ). $\mathrm{E}_{\mathrm{B}}$ at $1 \times 10^{9} / \mathrm{ml}$ were incubated with an equal volume of streptavidin $(250 \mu \mathrm{g} / \mathrm{ml})$ for $30 \mathrm{~min}$ at $4^{\circ} \mathrm{C}$ with mixing. The streptavidin-cbated $E_{B}\left(E_{B A}\right)$ were then washed three times with the buffer and resuspended to $1 \times 10^{9} \mathrm{E} / \mathrm{ml}$ for immediate use. Pooled hIgG or purified human myeloma proteins of specific IgG subclasses were biotinylated with NHS-LC-biotin ( $0.01 \mathrm{mg}$ biotin/mg protein) for $60 \mathrm{~min}$ at room temperature with occasional mixing. To bind the biotinylated hIgG to the $\mathrm{E}_{\mathrm{BA}}, \mathrm{E}_{\mathrm{BA}}\left(12.5 \mu \mathrm{l}\right.$ at $\left.1 \times 10^{9} / \mathrm{ml}\right)$ were combined with varying amounts of biotinylated protein $(200 \mu \mathrm{g} / \mathrm{ml}$ to $1 \mathrm{mg} / \mathrm{ml})$ for 30 min at $4^{\circ} \mathrm{C}$ with mixing. After three washes, the hIgG-coated $\mathrm{E}_{\mathrm{BA}}(\mathrm{E}$ $\mathrm{hIgG})$ were then resuspended in $125 \mu \mathrm{l}\left(1 \times 10^{8} \mathrm{E} / \mathrm{ml}\right)$ and used immediately. For certain experiments, the $\mathrm{E}_{\mathrm{BA}}$ were coupled to biotinylated IV.3 Fab. The results of experiments using E-IV.3 prepared by the biotin-avidin sandwich technique were comparable to those using EIV.3 Fab heteroantibodies (23-25).

Assay of phagocytosis. Quantitation of phagocytosis by PMNs and monocytes was performed as previously described $(10,21,26)$. Briefly, cells were resuspended in RPMI with $20 \%$ heat-inactivated IgG-free FCS (Gibco Laboratories), at $5 \times 10^{6}$ cells $/ \mathrm{ml}$. In certain experiments, the PMNs were preincubated with either IV.3 Fab or $3 \mathrm{G} 8 \mathrm{~F}\left(\mathrm{ab}^{\prime}\right)_{2}(30$ $\mu \mathrm{g} / \mathrm{ml}$ ) for $5 \mathrm{~min}$; the MAbs were present throughout the assay of phagocytosis. For experiments with FMLP-treated PMN $\left(10^{-7} \mathrm{M}\right)$, FMLP was added simultaneously with the phagocytic particle.

To assess internalization of $\mathrm{E}$ target particles, cells $(100 \mu \mathrm{l})$ were combined with EA $(250 \mu \mathrm{l})$, E-IV.3 $(250 \mu \mathrm{l})$, or E-hIgG $(125 \mu \mathrm{l})$. The leukocyte-erythrocyte mixtures were centrifuged at $44 \mathrm{~g}$ for $3 \mathrm{~min}$ and then incubated at $37^{\circ} \mathrm{C}$ for $15 \mathrm{~min}$ to allow for maximum internalization. After hypotonic lysis of noninternalized $\mathrm{E}$, phagocytosis was quantitated by light microscopy. At least 400 cells/slide were counted in duplicate without knowledge of the donor huFc $\gamma$ RIIA or huFc $\gamma$ RIIIB allotype. The data are expressed as phagocytic index (PI, number of ingested $\mathrm{E} / 100$ phagocytes). For assays with monocytes, PI was corrected for the percent peroxidase positivity of mononuclear cells.

Assay of adherence. To quantitate adherence, PMN and E target particles were prepared and combined as described in the assay of phagocytosis above. After centrifugation at $44 \mathrm{~g}$ for $3 \mathrm{~min}$, the PMN-E mixtures were maintained at room temperature for $10 \mathrm{~min}$ and then gently resuspended. Adherence of $\mathrm{E}$ to $\mathrm{PMN}$ was quantitated by light microscopy. Data are expressed as adherence index (AI, number of adherent or internalized erythrocytes/100 PMN). Under these conditions, $<1 \%$ of phagocytes had internalized $\mathrm{E}$.

Immunofluorescent flow cytometry. Fresh leukocytes $\left(5 \times 10^{5}\right.$ in PBS with $0.1 \%$ FCS) were incubated with saturating amounts of specific MAb or isotype controls for $30 \mathrm{~min}$ at $4^{\circ} \mathrm{C}$. After two washes with cold PBS containing $1 \%$ FCS, cells were incubated with saturating concentrations of phycoerythrin (PE)-conjugated goat anti-mouse IgG $\mathrm{F}\left(\mathrm{ab}^{\prime}\right)_{2}$ for $30 \mathrm{~min}$ at $4^{\circ} \mathrm{C}$ followed by washing twice with cold $\mathrm{PBS} / 1 \%$ 
FCS. E-hIgG were stained with FITC-conjugated rabbit anti-human IgG $\mathrm{F}\left(\mathrm{ab}^{\prime}\right)_{2}$, E coated with IV.3 Fab were stained with PE-conjugated goat anti-mouse IgG $\mathrm{F}\left(\mathrm{ab}^{\prime}\right)_{2}$, and $\mathrm{E}$ opsonized with rabbit anti-ox E antibodies were stained with FITC-conjugated goat anti-rabbit IgG $\mathrm{F}\left(\mathrm{ab}^{\prime}\right)_{2}$, followed by washes with cold PBS/1\% FCS.

After staining, cell-associated immunofluorescence was quantitated on a Cytofluorograf IIS (Becton Dickinson Immunocytometry Systems, Mountain View, CA) with a 2151 computer as previously described $(10,15,16)$. For each experiment, the instrument was calibrated with FITC-conjugated calf thymus nuclei (Fluorotrol-GF, Becton, Dickinson \& Co., Mountain View, CA) and quantitative PE microbead standards (Flow Cytometry Standards Corp., Research Triangle Park, NC) to allow for assessment of both absolute and relative levels of immunofluorescence.

Anti-CD3 proliferation assay. Peripheral blood mononuclear cells, separated by Ficoll-Hypaque as described above, were resuspended at 1 $\times 10^{6}$ cells/ml in RPMI 1640 with $10 \%$ FCS (supplemented with glutamine, penicillin, and streptomycin) and aliquoted into 96-well plates at $1 \times 10^{5}$ cells/well. OKT3 (IgG2a anti-CD3, $5 \mu \mathrm{g} / \mathrm{ml}$ final concentration), Leu4 (IgG1 anti-CD3, $5 \mu \mathrm{g} / \mathrm{ml}$ final concentration), nonspecific control isotype antibodies, or media alone were added to triplicate wells and the cells were incubated for $4 \mathrm{~d}$ at $37^{\circ} \mathrm{C}$. For the final $8 \mathrm{hr}$, $\left[{ }^{3} \mathrm{H}\right]$ thymidine (Amersham Corp., Arlington Heights, IL), $2 \mu \mathrm{Ci} /$ well was added, after which the cells were harvested, washed, and $\left[{ }^{3} \mathrm{H}\right]-$ thymidine incorporation measured in a beta counter (27-29).

Data analysis. For assessment of phagocytic capacity, all experiments were performed in a matched-pairs experimental design. Accordingly, each subject, homozygous for a given $\mathrm{Fc} \gamma \mathrm{R}$ isoform (huFc $\gamma$ RIIA or huFc $\gamma$ RIIIB), was studied in comparison to a second subject, homozygous for the other allele of the same isoform and matched for the same phenotype of the other Fc $\gamma$ R (e.g., HR-NA1 vs. LR-NA1).

Data are displayed as mean \pm SEM. PI for specific probes were compared using a paired $t$ test (two tailed). Differences in phagocytic capacity between the groups for $\mathrm{E}$ opsonized over a range of densities were compared by repeated measures analysis of variance (ANOVA). A probability of 0.05 was used to reject the null hypothesis that there is no difference between the groups.

\section{Results}

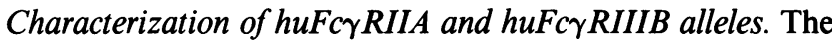
allotypic characteristics of our normal population for huFc $\gamma$ RIIIB were determined by serological typing and quantitative flow cytometry of PMN using NA1- and NA2-specific MAbs $(10,15,16)$. The LR-HR alleles of huFc $\gamma$ RIIA in our population were identified by quantitative flow cytometry with MAb 41H.16, which recognizes the HR allele of huFc $\gamma$ RIIA, and MAb IV.3, which recognizes both HR and LR alleles (17). Using the ratio of fluorescence intensity of $41 \mathrm{H} .16 / \mathrm{IV} .3$ in both monocytes and PMN, we assigned the homozygous HR phenotype to donors having a ratio of $0.88-1.1(n=8)$, the heterozygous HR/LR phenotype to donors having a ratio of 0.42-0.59 $(n=11)$, and the homozygous LR phenotype to donors having a ratio of $<0.13(n=13)$. The phenotypic assignment of homozygous individuals was corroborated by proliferation assays with anti-CD3 MAbs of both $\mathrm{mIgG1}$ and mIgG2a isotypes. In all cases, the results of the mitogenesis assays were in agreement with the flow cytometry assignment for huFc $\gamma$ RIIA.

Quantitative expression of huFc $\gamma$ RII as determined by flow cytometry using MAb IV.3 showed small differences between the phenotypes (PMN: HR 129 \pm 2 , HR/LR 128 \pm 3 , LR 121 \pm 2 mean channel log fluorescence units [MCF]; monocytes: HR $130 \pm 4$, HR/LR 122 \pm 3 , LR $118 \pm 4$ MCF; for PMN, HR vs. LR $P<0.02$, Mann-Whitney $U$ test; all other comparisons, $P$ $>0.1$ ). The expression of huFc $\gamma$ RIII as determined by flow cytometry using MAb 3G8, which recognizes the NA1 and NA2 alleles, was the same for all phenotypes (NA1 154 \pm 4 ; NA1/NA2 $150 \pm 2$; NA2 $152 \pm 6 ; P>0.1$ for all comparisons).

Phagocytosis by PMN from LR homozygotes is higher than $H R$ homozygotes. Given the difference in ligand binding by Fc $\gamma$ RIIA alleles transfected into fibroblasts or COS cells (7-9), we considered the possibility that the Fc $\gamma \mathrm{R}$-mediated phagocytic capacity of blood cells expressing these alleles might differ. Accordingly, we examined quantitative phagocytosis by PMN from normal donors who are homozygous for the LR or HR alleles. To control for differences due to huFc $\gamma$ RIIIB, each pair of homozygous donors was matched for NA alleles. Although not different when probed with anti-Fc $\gamma$ RII Fabcoated E (E-IV.3, a ligand-independent huFc $\gamma$ RII-specific probe), LR homozygotes showed significantly higher phagocytosis than HR homozygotes when probed with E-hIgG (PI [EhIgG internalized/100 PMN]: LR vs. HR, $50 \pm 7$ vs. $27 \pm 10, P$ $<0.003$; Fig. 1).

Over a broad range of opsonization densities, LR subjects had a greater level of phagocytosis of E-hIgG $(P<0.02$, repeated measures ANOVA). In contrast, phagocytosis EA and E-IV.3 prepared at a broad range of opsonization densities was indistinguishable between the two groups $(P>0.1$, repeated measures ANOVA). The absence of the difference in phagocytosis between LR and HR donors with EA and E-IV.3 suggested that the nature of the ligand might be important in defining the LR-HR functional difference.

Higher phagocytosis by FcrRIIA LR homozygotes: relationship to hIgG subclasses. Since the alleles of huFc $\gamma$ RIIA differentially bind murine $\operatorname{IgG} 1(9,27-32)$ and hIgG2 in a transfected fibroblast system (8), we examined the capacity of the LR-HR alleles expressed on blood cells to recognize and bind subclasses of hIgG. In each of seven pairs of homozygous LR and HR subjects matched for huFc $\gamma$ RIIIB alleles, there was a dramatic difference between the HR and LR individuals in

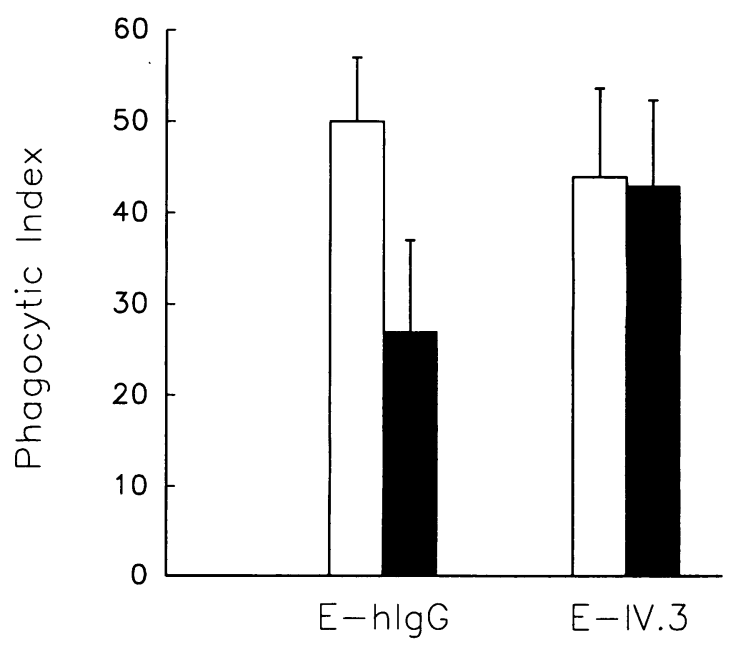

Figure 1. Phagocytosis of E-hIgG by PMNs from LR homozygotes is higher that by HR homozygotes. PMNs from normal donors, homozygous for the LR or HR alleles and matched for identical NA alleles of huFc $\gamma$ RIIIB, were studied simultaneously for internalization of E-IV.3 and E-hIgG in a matched-pairs design. PI was quantitated by light microscopy. In each of six pairs, internalization of E-IgG was greater for the homozygous LR subjects $(P<0.003$, paired $t$ test). In contrast, phagocytosis of E-IV.3 was indistinguishable between the two groups $(P=\mathrm{NS})$. (ㅁ) LR; ( $(\boldsymbol{)}) \mathrm{HR}$. 

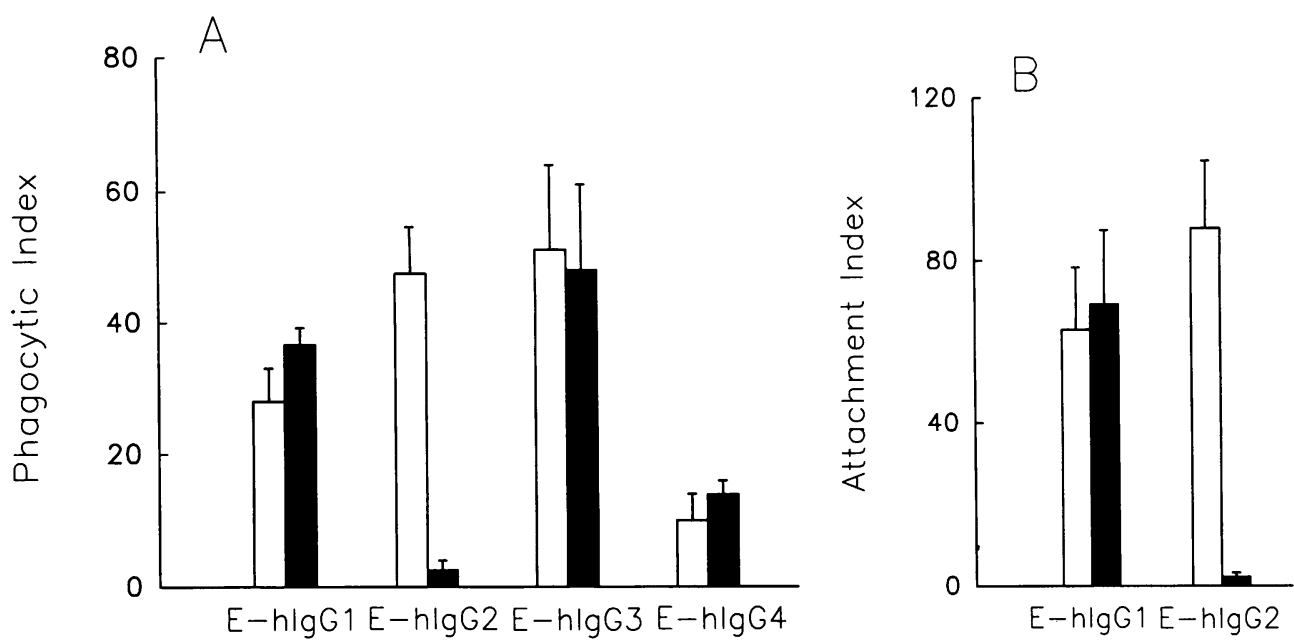

Figure 2. PMNs from LR homozygotes internalize E-hIgG2. PMNs from pairs of homozygous HR and LR donors with identical NA alleles were studied simultaneously with all probes. $(A)$ For the assay of phagocytosis, PMNs were incubated with EhIgG1, E-hIgG2, E-hIgG3, or EhIgG4 opsonized with similar intermediate densities of IgG (MCF 150-160). In each of seven pairs, there was minimal internalization of E-hIgG2 by HR donors ( $L R$ vs. $H R, P$ $<0.0004)$. No significant difference in phagocytosis of the other probes was noted. $(B)$ For the assay of attachment, PMNs were incubated with E-hIgG1 or E-hIgG2. In each of six pairs studied, there was minimal binding of E-hIgG2 to HR PMNs ( $L R$ vs. $H R, P<0.003$ ), whereas binding of E-hIgG1 was similar for LR and HR. (口) LR; (घ) HR.

phagocytosis of E-hIgG2 (PI [E internalized/100 PMN]: LR vs. HR, $47 \pm 7$ vs. $3 \pm 1, P<0.0004$ ), whereas internalization of E-hIgG1, E-hIgG3, and E-hIgG4 was similar for both donor types (Fig. $2 \mathrm{~A}$ ). The lack of internalization of E-hIgG2 reflected an inability of the E-hIgG2 to bind to PMNs from HR subjects (AI for $\mathrm{E}$ with intermediate density of opsonization: 0-3 E attached/100 PMN; Fig. 2 B). Identical results were obtained with a second and different myeloma preparation of each hIgG subclass. A series of blocking experiments confirmed that huFc $\gamma$ RII was responsible for the phagocytosis of E-hIgG2. Pretreatment of PMN with MAb IV.3 Fab $(30 \mu \mathrm{g} / \mathrm{ml})$ to block huFc $\gamma$ RII completely abrogated internalization of EhIgG2 (Fig. 3). Indeed, pretreatment with $3 \mathrm{G} 8 \mathrm{~F}\left(\mathrm{ab}^{\prime}\right)_{2}$ to block huFc $\gamma$ RIIIB did not block, but rather enhanced, phagocytosis of E-hIgG2 consistent with our previous findings that crosslinking huFc $\gamma$ RIIIB primes phagocytosis mediated by huFc $\gamma$ RII in PMN (PI: control vs. $3 \mathrm{G} 8 \mathrm{~F}\left(\mathrm{ab}^{\prime}\right)_{2}, 47 \pm 16 \mathrm{E}$ internalized/100 PMN vs. $72 \pm 16, P>0.05)(25)$.

The capacity of the LR allele of huFc $\gamma$ RIIA to bind mIgG1 and form subclass-specific rosettes or induce $\mathrm{T}$ cell proliferation $(32,33)$ is dependent in part on the concentration of MAb presented to receptor-bearing cells. A similar relationship is apparent for the capacity of the HR allele of huFc $\gamma$ RIIA on PMN to bind hIgG2. As shown in Figure $4 A$, higher densities of hIgG2 on E resulted in a higher PI in both LR and HR donors. At all levels of hIgG2, however, a marked difference between the LR and HR groups was evident $(P<0.001$, repeated measures ANOVA). In contrast, the phagocytosis of E-hIgGl showed no difference between the two groups at any level of E surface hIgG1 (Fig. $4 \mathrm{~B}$ ). Similarly, there was no difference in internalization of E-hIgG4 opsonized over a range of densities between both groups. For example, the PI for EhIgG4 with MCF 170-179 was $29 \pm 10 \mathrm{E}$ internalized/100 PMN for LR donors and $27 \pm 6$ for HR donors.

Interestingly, internalization of E-hIgG and E-hIgG1 by HR PMN was identical over most of the range of opsonization, whereas internalization of E-hIgG by LR PMN was higher than that of E-hIgG1 with similar densities of opsonization. This difference is presumably due to recognition of $\mathrm{hIgG} 2$ on the E-hIgG by the LR allele, and this observation suggests that functional differences between the huFc $\gamma$ RIIA alleles may be apparent even with polyclonal antibody responses.
Detection of the differential capacity of huFc $\gamma$ RIIA alleles to bind hIgG2 is not restricted to PMNs. Human monocytes, which express both Fc $\gamma$ RIIA and Fc $\gamma$ RI, show efficient internalization of E-hIgG2 when derived from LR subjects but little internalization when derived from $H R$ subjects (PI [E internalized/100 monocytes]: $L R$ vs. $H R, 145 \pm 14$ vs. $21 \pm 3, P$ $<0.0005$ ). In contrast, phagocytosis of both E-hIgG and EhIgG1 was comparable for both groups (PI [E internalized/100 monocytes]: $L R$ vs. HR, $152 \pm 10$ vs. $146 \pm 25$ and $149 \pm 13$ vs. $153 \pm 37$, respectively, $P>0.1$ ). Blockade of huFc $\gamma$ RII with IV.3 Fab blocked internalization of E-hIgG2 by monocytes in LR subjects (PI [E internalized/100 monocytes]: $169 \pm 43$ vs. $10 \pm 6, P<0.03$ ), similar to the findings in PMN (Fig. 3).

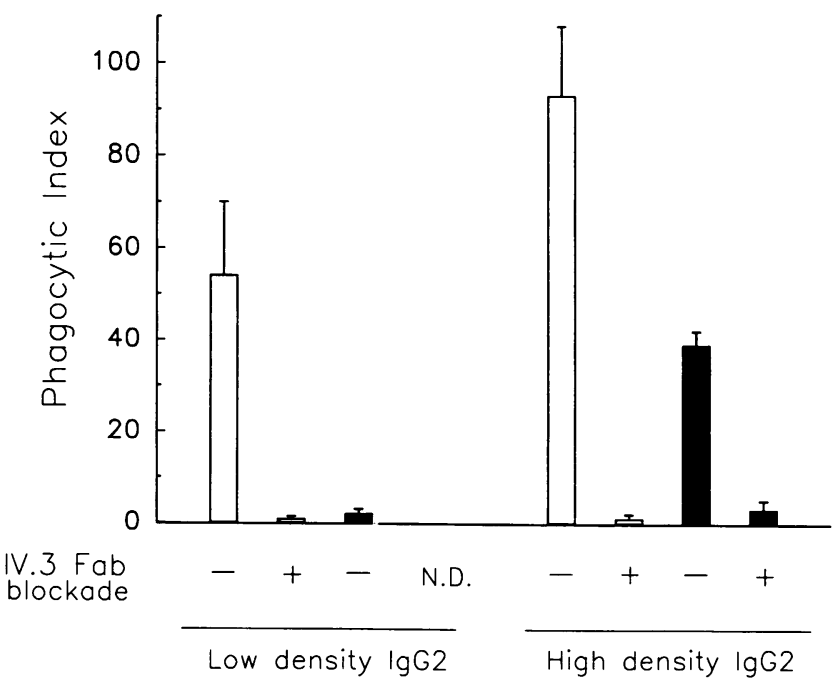

Figure 3. Blockade of huFc $\gamma$ RIIA with IV.3 Fab completely inhibits internalization of E-hIgG2 in both LR and HR PMNs. PMNs from $\mathrm{HR}$ and LR donors were pretreated with IV. $3 \mathrm{Fab}(30 \mu \mathrm{g} / \mathrm{ml})$ or control medium for $10 \mathrm{~min}$ and then combined with E-hIgG2 prepared at lower (intermediate density; MCF 150-160) or higher (MCF 170180) density opsonization. In each experiment both an HR and an LR donor were studied simultaneously ( $n=3-4$ pairs). Pretreatment of PMN with IV.3 Fab blocked phagocytosis of both E-hIgG2 probes in LR donors (control vs. IV.3 Fab, $P<0.03$ ). In HR donors, IV.3 Fab blocked phagocytosis of the high density E-hIgG2 (control vs. IV.3 Fab, $P<0.006$ ). (ㅁ) LR; (ロ) HR. 

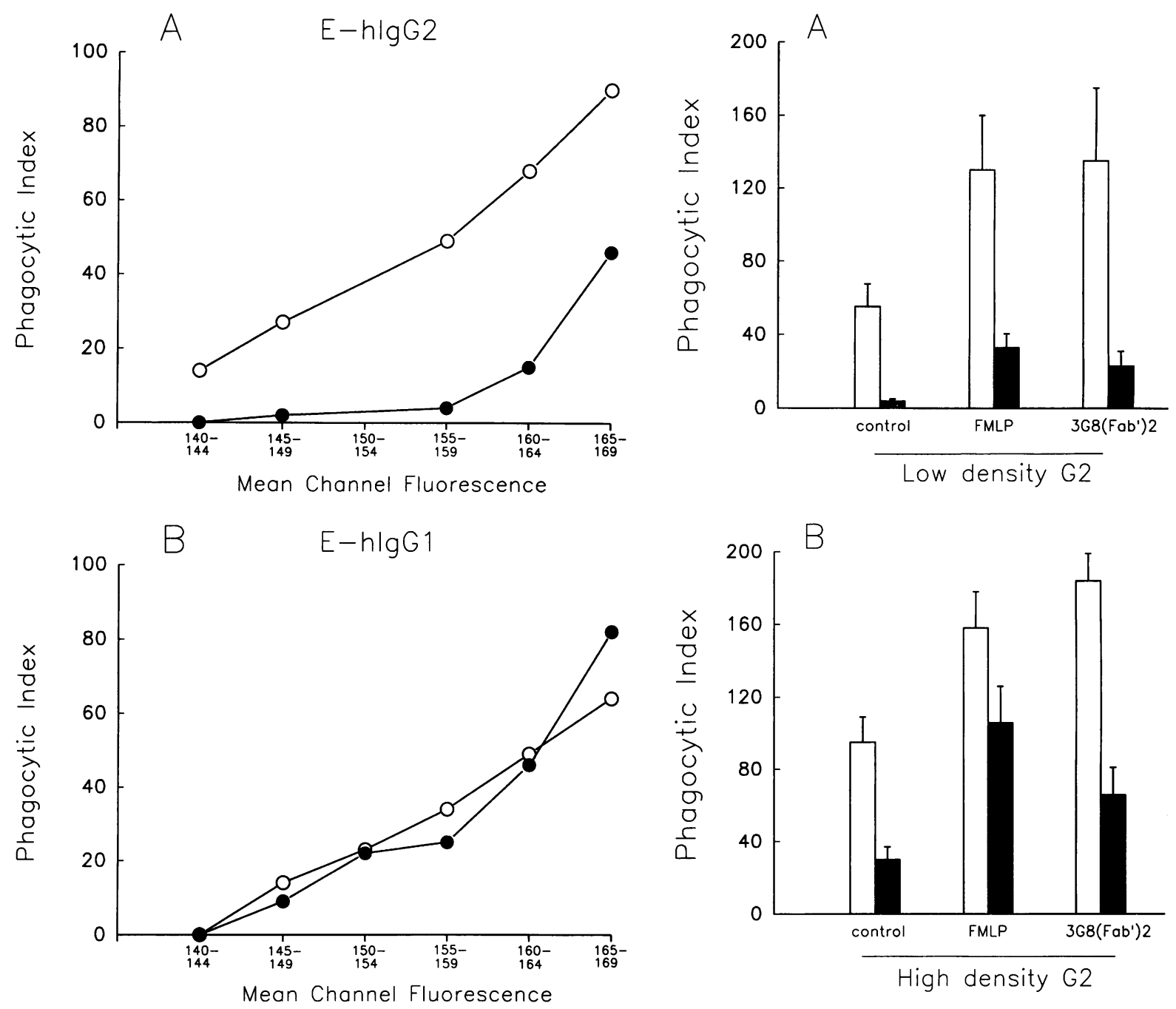

Figure 4. The lack of internalization of E-hIgG2 by PMNs from homozygous HR subjects is relative, not absolute. PMNs from HR and LR were combined with $\mathrm{E}$ opsonized with varying amounts of hIgG2 $(A)$ or hIgG1 $(B)$, as determined by flow cytometry $(n=3-5$ pairs/level). Phagocytosis of all probes was directly related to degree of opsonization. $(A)$ Internalization of E-hIgG2 was higher for LR donors at all levels of opsonization $(P<0.001$, repeated measures ANOVA), $(B)$ whereas that for E-hIgG1 remained similar for both groups. (O) LR; (•) HR.

The difference in $E$-hIgG2 internalization between LR and $H R$ donors persists in primed PMNs. Simultaneous engagement of several receptors may be the most relevant physiological stimulus for phagocytes. For example, it is unlikely that microbes opsonized in vivo or endogenously formed immune complexes would be composed exclusively of hIgG2 and thus ligate only huFc $\gamma$ RII. The observation that treatment of PMNs with $3 \mathrm{G} 8 \mathrm{~F}\left(\mathrm{ab}^{\prime}\right)_{2}$ to crosslink huFc $\gamma$ RIIIB augmented internalization of E-hIgG2 raised the possibility that primed or activated PMNs found in sites of infection or inflammation might not show a difference between $L R$ and HR donors. To examine this possibility, PMNs from LR and HR donors were pretreated with $3 \mathrm{G} 8 \mathrm{~F}\left(\mathrm{ab}^{\prime}\right)_{2}$, FMLP, or control medium. In spite of an increase in E-hIgG2 internalization, phagocytosis of EhIgG 2 by LR PMNs remained significantly higher than that by HR PMNs (Fig. 5). The increase in E-hIgG2 phagocytosis was associated with an increase in E-hIgG2 binding in both LR and

Figure 5. The difference in E-hIgG2 internalization between LR and HR donors persists in primed PMN. PMN from HR and LR donors were treated with FMLP $\left(10^{-7} \mathrm{M}\right), 3 \mathrm{G} 8 \mathrm{~F}\left(\mathrm{ab}^{\prime}\right)_{2}(30 \mu \mathrm{g} / \mathrm{ml})$, or control medium before quantitation of phagocytosis of E-hIgG2 prepared at lower (intermediate density; MCF 150-160) and higher (MCF 170$180)$ density opsonization. Phagocytosis of the $(A)$ lower density $E$ hIgG2 $(A)$ and the $(B)$ higher density E-hIgG2 was greater for LR donors $(n=3-9)$. Low density E-hIgG2, LR vs. HR: control $P<0.01$, FMLP $P<0.03$, 3G8 F(ab') $P<0.01$; High density E-hIgG2, LR vs. HR: control $P<0.001$, FMLP $P<0.03,3 \mathrm{G} 8 \mathrm{~F}\left(\mathrm{ab}^{\prime}\right)_{2} P<0.03$. () LR; (ロ) HR.

HR donors, although this may not be only mechanism for the increase in internalization (AI for LR [E-hIgG2 adherent/100 PMN]: control 134 \pm 34 , FMLP $219 \pm 36,3 \mathrm{G8} \mathrm{F}\left(\mathrm{ab}^{\prime}\right)_{2} 150 \pm 42$, $n=5-6$, FMLP vs. control $P<0.02$; AI for HR: control $8 \pm 3$, FMLP $72 \pm 15,3 \mathrm{G} 8 \mathrm{~F}\left(\mathrm{ab}^{\prime}\right)_{2} 40 \pm 10 n=3-4$, FMLP vs. control $P$ $<0.04)$. Phagocytosis of E-hIgG2 by FMLP-stimulated PMNs from homozygous HR donors was also completely inhibited by blockade of huFc $\gamma$ RII with IV.3 Fab (PI [E internalized/100 PMN]: FMLP-treated PMNs $=37 \pm 9$, FMLP-treated PMNs in the presence of IV.3 Fab $=5 \pm 2 ; n=4 ; P<0.05$ ). Thus, in primed PMNs, hIgG2 binding and internalization is predominantly mediated by huFc $\gamma$ RII.

Higher phagocytic capacity of PMNs from NA1 subjects is not related to allelic polymorphisms of huFc $\gamma$ RIIA. Homozygous NA1 donors have higher phagocytosis of EA than homo- 
zygous NA2 donors, which is Fc $\gamma \mathrm{R}$ specific rather than due to differences in generalized phagocytic capacity (10). Given the differences in Fc $\gamma$ RIIA-mediated phagocytosis and the recent data localizing the genes for both huFc $\gamma$ RII and huFc $\gamma$ RIII to human chromosome 1 (11-13), we determined whether an association between the alleles of huFc $\gamma$ RIIA and the NA1-NA2 alleles of huFc $\gamma$ RIIIB might explain the higher phagocytic capacity of NA1 homozygotes. In each of six pairs of NA1 and NA2 homozygous individuals, each with identical huFc $\gamma$ RIIA alleles, internalization of EA and of E-hIgG was higher in the NA1 donor, independent of the huFc $\gamma$ RIIA phenotype of the donor pair (Fig. 6). The difference in phagocytic capacity was not related to alterations in binding of E-hIgG (AI for standard level of opsonization [E-hIgG adherent/100 PMN]: NA1 vs. NA2 $=265 \pm 69$ vs. $280 \pm 66, n=5$ ). Simultaneous analysis of huFc $\gamma$ RII-specific phagocytosis in the NA1 and NA2 homozygous donors with E-IV.3 showed no significant differences (PI [E internalized/100 PMN]: NA1 vs. NA2 $=32 \pm 12$ vs. $29 \pm 10$, $n=5$; Fig. 6). Thus, an huFc $\gamma$ RIIIB-specific mechanism, unrelated to the quantitative binding of ligand and unrelated to huFc $\gamma$ RIIA alleles, appears to be responsible for the difference in phagocytosis between NA1 and NA2 homozygous donors.

\section{Discussion}

Allelic polymorphisms of human Fc $\gamma \mathrm{R}$ have functionally distinct capacities that are important in mediating interactions between human phagocytes and IgG ligands. Using E-hIgG, we show consistently higher PMN phagocytosis by individuals homozygous for the LR allele of huFc $\gamma$ RIIA and by individuals homozygous for the NA1 allele of huFc $\gamma$ RIIIB. This difference

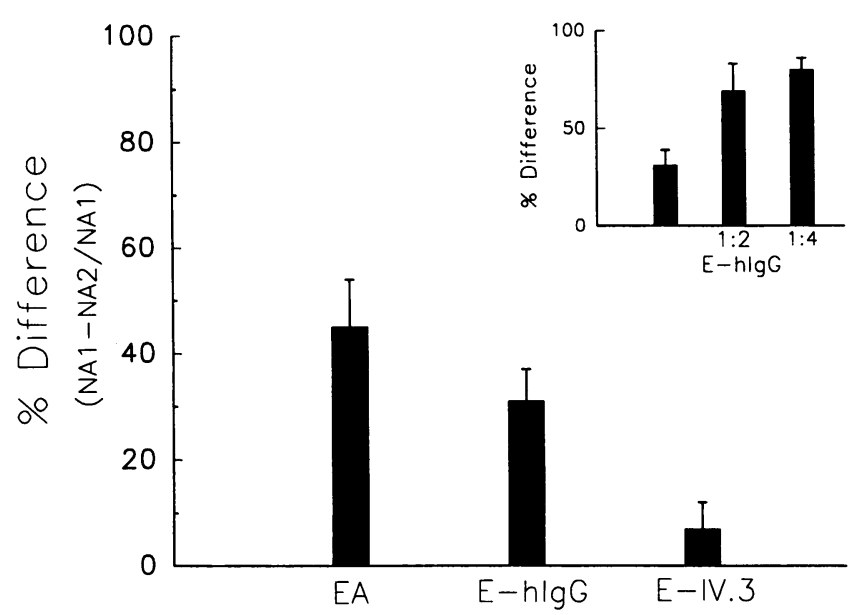

Figure 6. Fc $\gamma$ R-mediated phagocytosis of EA and E-hIgG, but not E-IV.3, is higher in homozygous NA1 subjects compared with that of NA2 subjects. PMN from NA1 and NA2 homozygous donors with identical HR-LR alleles were assayed simultaneously with EA, EhIgG, and E-IV.3, and the percent difference between the donors was calculated. In each of six pairs studied, the NA2 donor had a lower phagocytic capacity for EA and E-hIgG (PI for EA: 45 $\pm 9 \%$ lower, $P$ $<0.03$; PI for E-hIgG: $33 \pm 8 \%$ lower, $P<0.003)$. Phagocytosis of E-IV.3 was similar for both groups $(P=\mathrm{NS})$. As with EA (9), the difference in phagocytosis of E-hIgG became more pronounced with less opsonization of the $\mathrm{E}$, such that a fourfold dilution of the standard concentration of hIgG showed an $80 \pm 10 \%$ difference in PI (insert). The dilutions of biotinylated hIgG used to opsonize $\mathrm{E}_{\mathrm{BA}}$ corresponded to MCFs of 164 (std), 158 (1:2), and 144 (1:4). is even more pronounced in LR homozygous subjects for hIgG2 subclass-specific phagocytosis, since, in contrast to $H R$, the LR allele of huFc $\gamma$ RIIA binds hIgG2 efficiently.

The differences in functional capacity for the two alleles of huFc $\gamma$ RIIA appear to be mediated by a mechanism distinct from that involved in the differences between the two alleles of huFc $\gamma$ RIIIB. The biallelic HR-LR polymorphism of huFc $\gamma$ RIIA alters the affinity of the ligand binding site for some subclasses of IgG ligand. Originally distinguished by differences in binding of $\mathrm{mIgG} 1$ to human monocytes as assessed by $\mathrm{T}$ cell proliferation induced by anti-CD3 MAb (27-32), the functional HR-LR phenotype is determined by a single amino acid present at position 131 , which plays a critical role in the binding capacity for $\operatorname{mIgG1}(6,7,9)$. Warmerdam and colleagues (8) also showed that fibroblast transfectants expressing the LR allele (histidine residue at position 131) lack the capacity to bind $\mathrm{mIgG1}$ but can bind hIgG2, whereas transfectants expressing the HR allele (arginine residue at position 131) bind mIgG1 but do not recognize hIgG2. Importantly, the functional impact of this difference is not restricted to transfected fibroblasts expressing a single receptor family but is evident in resting and activated human phagocytes. Our data indicate that for human PMN, which express both huFc $\gamma$ RII and huFc $\gamma$ RIIIB, submaximally opsonized E-hIgG are internalized more efficiently by LR donors. This difference reflects the ability of LR donors to bind hIgG2, which comprises $~ 20 \%$ of pooled hIgG $(34,35)$. E-hIgG2 are internalized efficiently by individuals homozygous for LR allele of huFc $\gamma$ RIIA but are internalized poorly by individuals homozygous for the HR allele (Fig. 2). Even in "primed" PMNs, which demonstrate enhanced binding and internalization of E-hIgG2, the difference between HR and LR donors persists (Fig. 5). This difference in E-hIgG2 phagocytosis is also evident in human monocytes that express both huFc $\gamma$ RI and huFc $\gamma$ RII. The inability of HR PMN to bind hIgG2 is relative rather than absolute, and at high levels of opsonization there is a modest degree of internalization of E-hIgG2 (Fig. 4). A similar discriminative preference for the other human subclasses of IgG was not identifiable. Although absolute comparisons of efficiency of binding and internalization for each of the hIgG subclasses are not possible, in part because determinations of "comparable densities" of opsonization may be influenced by unique properties of individual myeloma proteins and by properties of the second antibody, our results suggest that in LR PMN the relative efficiency is hIgG1 = hIgG3 = hIgG2 > hIgG4 and, in HR PMN, hIgG1 $=\mathrm{hIgG} 3>\mathrm{hIgG} 4>\mathrm{hIgG} 2$.

The biallelic NA1-NA2 polymorphism of huFc $\gamma$ RIIIB, expressed only on human PMNs, is related to several amino acid differences, two of which alter potential N-linked glycosylation sites (1-5). Since huFc $\gamma$ RIIIB may function in part as a binding molecule to present ligand to huFc $\gamma$ RIIA (24), we considered the contribution of the LR-HR alleles of huFc $\gamma$ RIIA to the functional consequences of the huFc $\gamma$ RIIIB polymorphism. The current results demonstrate higher phagocytosis of E-hIgG by NA1 donors compared with NA2 donors, despite matching of donors for huFc $\gamma$ RIIA alleles (Fig. 6) and no difference in phagocytosis of E-IV.3 (an huFc $\gamma$ RII-specific probe) by NAl and NA2 donors (Fig. 6). These data, along with our previous observation that blockade of huFc $\gamma$ RII with IV.3 Fab amplifies the difference in phagocytosis between NA1 and NA2 (10), support the conclusion that the difference in EA and E-hIgG phagocytosis between NA1 and NA2 homozygous donors is 
independent of huFc $\gamma$ RIIA phenotype. The mechanism underlying the difference between NA1 and NA2 homozygotes remains undefined, although IgG-independent, allele-specific interactions with other surface membrane molecules provide provocative possibilities.

The implications of these two allotypic systems are intriguing. The NA1-NA2 functional polymorphism appears to be IgG-ligand independent. No differences in opsonized target binding have been demonstrated, and no subclass preference has been identified since, among HR homozygous donors, the inability to bind $\mathrm{hIgG} 2$ is equally apparent for both the NA1 homozygous and NA2 homozygous phenotypes. The difference in internalization is evident with concanavalin-treated $\mathrm{E}$, which engage huFc $\gamma$ RIIIB through nonclassical (IgG-ligand independent) carbohydrate-mediated interactions (10). The fact that the NA1-NA2 alleles are functionally distinct indicates, however, that huFc $\gamma$ RIIIB serves as an integral participant in phagocytosis by PMNs. In contrast, the HR-LR functional polymorphism is directly ligand dependent, and the ability of LR PMNs and monocytes to bind hIgG2 efficiently suggests that a reexamination of the role of hIgG2 humoral response may be appropriate. For example, since the antibody response to bacterial polysaccharide antigens is predominantly hIgG2 isotype (36), the relationship between $\mathrm{hIgG} 2$ levels and susceptibility to infection with encapsulated bacterial pathogens (34, $35,37)$ may be influenced by the HR-LR phenotype of the host. Thus, the increased risk of invasive Hemophilus influen$z a e$ infection in certain populations that have impaired antibody responses to $H$. influenzae and low hIgG 2 levels $(38,39)$ may be greater for HR homozygotes than for LR homozygotes. Similarly, in cystic fibrosis, where bacterial colonization of the lungs by Pseudomonas aeruginosa is associated with high serum levels of hIgG2 anti-Ps. aeruginosa antibodies and immune complexes containing hIgG2 anti-Ps. aeruginosa antibodies, the potential for the hIgG2 antibodies to act as poor opsonins and inhibit clearance of the organisms (40-42) may vary in accord with the HR-LR phenotype of the host. Importantly, activation of PMNs does not abrogate this allelic difference in function. Indeed, one might speculate that the evolutionary pressure for the presence of the LR allele of huFc $\gamma$ RIIA (29) is related to more effective defense against microbial agents eliciting an hIgG2 humoral response.

The precedent for allelic differences in receptor structure having a significant impact on phagocyte function is of potential importance given the structural diversity of huFc $\gamma R$. Definition of further such structure-function relationships among huFc $\gamma \mathrm{R}$ may provide insights into both disease susceptibility and pathogenesis.

\section{Acknowledgments}

We greatly appreciate the participation of the normal volunteers without whose assistance these studies could not have been completed. We thank Dr. Mary Crow for performing the anti-CD3 proliferation assays; Carl Triscari for assistance with flow cytometry; Drs. Jan G. J. van de Winkel, Petra A. M. Warmerdam, and Allan Gibofsky for useful discussions; Dr. Margaret Peterson for assistance in statistical analysis; and Dr. Charles L. Christian for his continued support.

This work was supported in part by grants RO1-AR38889 (Dr. Salmon) and RO1-AR33062 (Dr. Kimberly) awarded by the National Institutes of Health. Dr. Edberg is a Fellow of the Irvington Institute for Medical Research. The Flow Cytometry Core Facility at The Hospital for Special Surgery and the Research Methodology Core (Biometry) were supported in part by the Cornell Multipurpose Arthritis and Musculoskeletal Diseases Center (P60-AR38520).

\section{References}

1. Werner, G., A. E. G. Kr. von dem Borne, M. J. E. Bos, J. F. Tromp, C. M. van der Plas-van Dalen, F. J. Visser, C. P. Engelfriet, and P. A. Tetteroo. 1985. Localization of the human NA1 alloantigen on neutrophils to $\mathrm{Fc} \gamma$ receptors. In Leucocyte Typing II. Vol. 3. E. L. Reinherz, B. F. Haynes, L. M. Nadler, and I. D. Bernstein, editors. Springer-Verlag, New York, 109-121.

2. Tetteroo, P. A. T., C. E. Van der Schoot, F. J. Visser, M. J. E. Bos, and A. G. E. Kr. von dem Borne. 1987. Three different types of FC $\gamma$ receptors on human leukocytes defined by workshop antibodies: $\mathrm{Fc} \gamma \mathbf{R}_{\text {bow }}$ of neutrophils, $F_{c} \gamma R_{\text {low }}$ of NK/K lymphocytes and Fc $\gamma$ RII. In Leucocyte Typing III. A. J. McMichael, editor. Oxford University Press, Oxford, UK. 702-706.

3. Ory, P., I. M. Goldstein, E. E. Kwoh, and S. Clarkson. 1989. Characterization of polymorphic forms of Fc $\gamma$ RIII on human neutrophils. J. Clin. Invest. 83:1676-1681.

4. Ory, P., M. R. Clark, E. E. Kwoh, S. B. Clarkson, and I. M. Goldstein. 1989. Sequences of complementary DNAs that encode the NA1 and NA2 forms of Fc $\gamma$ receptor III on neutrophils. J. Clin. Invest. 84:1688-1691.

5. Ravetch, J. V., and B. Perussia. 1989. Alternative membrane forms of Fc $\gamma$ RIII (CD16) on human natural killer cells and neutrophils. Cell type-specific expression of two genes that differ in single nucleotide substitutions. J. Exp. Med. 170:481-497.

6. Clark, M. R., S. B. Clarkson, P. A. Ory, N. Stollman, and I. M. Goldstein. 1989. Molecular basis for a polymorphism involving $\mathrm{Fc}$ receptor II on human monocytes. J. Immunol. 143:1731-1734.

7. Warmerdam, P. A. M., J. G. J. van de Winkel, E. J. Gosselin, and P. J. A. Capel. 1990. Molecular basis for a polymorphism of human Fc $\gamma$ receptor II (CD32). J. Exp. Med. 172:19-25.

8. Warmerdam, P. A. M., J. G. J. van de Winkel, A. Vlug, N. A. C. Westerdal, and P. J. A. Capel. 1991. A single amino acid in the second Ig-like domain of the human $\mathrm{Fc} \gamma$ receptor II is critical for human IgG2 binding. J. Immunol. 147:1338-1343.

9. Clark, M. R., S. G. Stuart, R. P. Kimberly, P. A. Ory, and I. M. Goldstein. 1991. A single amino acid distinguishes the high-responder from low-responder form of Fc receptor II on human monocytes. Eur. J. Immunol. 21:1911-1916.

10. Salmon, J. E., J. C. Edberg, and R. P. Kimberly. 1990. Fc $\gamma$ receptor III on human neutrophils. Allelic variants have functionally distinct capacities. J. Clin. Invest. 85:1287-1295.

11. Peltz, G. A., H. O. Grundy, R. V. Lebo, H. Yssel, G. S. Barsh, and K. W. Moore. 1989. Human FcrRIII: cloning, expression, and identification of the chromosomal locus of two Fc receptors for IgG. Proc. Natl. Acad. Sci. USA. 86:1013-1017.

12. Grundy, H. O., G. Peltz, K. W. Moore, M. S. Golbus, M. L. G. Jackson, and $R$. V. Lebo. 1989. The polymorphic Fc $\gamma$ receptor II gene maps to the human chromosome 1q. Immunogenetics. 29:331-339.

13. Brooks, D. G., W. Q. Qiu, A. D. Luster, and J. V. Ravetch. 1989. Structure and expression of human IgG FcRII (CD32). Functional heterogeneity is encoded by the alternatively spliced products of multiple genes. J. Exp. Med. 170:1369-1385.

14. Lalezari, P. 1977. Neutrophil antigens: immunology and clinical implications. In The Granulocyte: Function and Clinical Utilization. T. Greenwalt and G. S. Jamieson, editors. Alan R. Liss, New York, 209-225.

15. Edberg, J. C., P. B. Redecha, J. E. Salmon, and R. P. Kimberly. 1989. Human FcyRIII (CD16). Isoforms with distinct allelic expression, epitopes displays and membrane anchors on PMN and NK cells. J. Immunol. 143:16421649.

16. Edberg, J. C., M. Barinsky, P. B. Redecha, J. E. Salmon, and R. P. Kimberly. 1990. Fc $\gamma$ RIII expressed on cultured monocytes is an N-glycosylated transmembrane protein. Similarity to Fc $\gamma$ RIII expressed on NK cells. J. Immunol. 144:4729-4734.

17. Gosselin, E. J., M. F. Brown, C. L. Anderson, T. F. Zipf, and P. M. Guyre. 1990. The monoclonal antibody $41 \mathrm{H} 16$ detects the Leu 4 responder form of human FcyRII. J. Immunol. 144:1817-1822.

18. Fleit, H. B., S. D. Wright, and J. C. Unkeless. 1982. Human neutrophil Fc $\gamma$ receptor distribution and structure. Proc. Natl. Acad. Sci. USA. 79:32753279.

19. Looney, R. J., D. H. Ryan, K. Takahashi, H. B. Fleit, H. J. Cohen, G. N. Abraham, and C. L. Anderson. 1986. Identification of a second class of IgG Fc receptors on human neutrophils. A 40 kilodalton molecule also found on eosinophils. J. Exp. Med. 163:826-836.

20. Antoun, G. R., B. M. Longenecker, and T. F. Zipf. 1989. Comparison of the $40 \mathrm{kDa}$ hematopoietic cell antigens bound by monoclonal antibodies IV.3, $41 \mathrm{H} .16$ and KB61. Mol. Immunol. 26:333-338.

21. Salmon, J. E., S. Kapur, and R. P. Kimberly. 1987. Opsonin-independent ligation of $F \mathrm{C} \gamma$ receptors: the 3G8-bearing receptors on neutrophils mediate the 
phagocytosis of concanavalin A-treated erythrocytes and nonopsonized $E$. coli. J. Exp. Med. 166:1798-1813.

22. Edberg, J. C., and R. P. Kimberly. 1992. Receptor specific probes for the study of $\mathrm{Fc} \gamma$ receptor specific function. J. Immunol. Methods. In press.

23. Shen, L., P. M. Guyre, C. L. Anderson, and M. W. Fanger. 1986. Heteroantibody-mediated cytotoxicity: antibody to the high affinity Fc receptor for IgG mediates cytotoxicity by human monocytes that is enhanced by interferongamma and is not blocked by human IgG. J. Immunol. 137:3378-3382.

24. Anderson, C. L., L. Shen, D. M. Eicher, M. D. Wewers, and J. K. Gill. 1990. Phagocytosis mediated by three distinct $\mathrm{Fc} \gamma$ receptor classes on human leukocytes. J. Exp. Med. 171:1333-1345.

25. Salmon, J. E., N. L. Brogle, J. C. Edberg, and R. P. Kimberly. 1991. Fc $\gamma$ receptor III induces actin polymerization in human neutrophils and primes phagocytosis mediated by Fc $\gamma$ receptor II. J. Immunol. 146:997-1004.

26. Salmon, J. E., R. P. Kimberly, A. Gibofsky, and M. Fotino. 1986. Altered phagocytosis by monocytes from HLA-DR2 and DR3-positive healthy adults is Fc $\gamma$ receptor-specific. J. Immunol. 136:3625-3630.

27. Tax, W. J. M., H. W. Willems, P. P. M. Reekers, P. J. A. Capel, and R. A. P. Koene. 1983. Polymorphism in mitogenic effect of $\mathrm{IgG} 1$ monoclonal antibodies against T3 antigen on human T cells. Nature (Lond.). 304:445-447.

28. Tax, W. J. M., F. F. M. Hermes, R. W. Willems, P. J. A. Capel, and R. A. P. Koene. 1984. Fc Receptors for mouse IgG1 on human monocytes: polymorphism and role in antibody-induced T cell proliferation. J. Immunol. 133:1185-1189.

29. Abo, T., A. B. Tilden, C. M. Balch, K. Kumagai, G. M. Troup, and M. D. Cooper. 1984. Ethnic differences in the lymphocyte proliferative response induced by murine IgG1 antibody, Leu 4, to the T3 molecule. J. Exp. Med. 160:303-309.

30. Cueppens, J. L., and F. van Vaeck. 1987. Direct demonstration of binding of anti-Leu 4 antibody to the $40 \mathrm{kDa} \mathrm{Fc}$ receptor on monocytes as a prerequisite for anti-Leu 4 induced T cell mitogenesis. J. Immunol. 139:4067-4071.

31. van de Winkel, J. G. J., G. J. J. C. Boonen, P. L. W. Janssen, A. Vlug, N. Hoog, and W. J. M. Tax. 1989. Activity of two types of Fc receptors, Fc $\gamma$ RI and Fc $\gamma$ RII, in human monocyte cytotoxicity to sensitized erythrocytes. Scand. $J$. Immunol. 29:23-31.
32. Clement, L. T., A. B. Tilden, and N. E. Dunlap. 1985. Analysis of the monocyte $\mathrm{Fc}$ receptor and antibody-mediated cellular interactions required for the induction of T cell proliferation by anti-T3 antibodies. J. Immunol. 135:165171.

33. Boot, J. H. A., M. E. J. Geerts, and L. A. Aarden. 1989. Functional polymorphisms of $\mathrm{Fc}$ receptors in human monocyte-mediated cytotoxicity towards erythrocytes induced by murine isotype switch variants. J. Immunol. 142:1217-1222.

34. Schur, P. H. 1987. IgG subclasses-a review. Ann. Allergy. 58:89-99.

35. Jefferis, R., and D. S. Kumararatne. 1990. Selective IgG subclass deficiency: quantification and clinical relevance. Clin. Exp. Immunol. 81:357-367.

36. Yount, W. J., M. M. Corner, H. G. Kunkel, and E. A. Kabat. 1968. Studies on human antibodies. VI. Selective variations in subgroup composition and genetic marker. J. Exp. Med. 127:633-646.

37. Scott, M. G., D. E. Briles, and M. H. Nahm. 1990. Selective IgG subclass expression: biologic, clinical and functional aspects. In The Human IgG Subclasses: Molecular Analysis of Structure, Function and Regulation. F. Shakib, editor. Pergamon Press, Oxford, UK. 161-183.

38. Siber, G. R., M. Santosham, G. R. Reid, C. Thompson, J. Almeido-Hill, A. Morell, G. deLange, J. K. Ketchum, and E. H. Callahan. 1990. Impaired antibody response to Haemphilus influenza type b polysaccharide and low IgG2 and IgG4 concentrations in Apache children. N. Engl. J. Med. 323:1387-1392.

39. Ward, J. G. Brenneman, G. W. Letson, W. L. Heyward, and the Alaska $H$. influenza Vaccine Study Group. 1990. Limited efficacy of a Haemophilus influenza type b conjugate vaccine in Alaska infants. N. Engl. J. Med. 323:1393-1401.

40. Fick, R. B., Jr., J. Olchowski, S. U. Squier, W. W. Merrill, and H. Y. Reynolds. 1986. Immunoglobulin-G subclasses in cystic fibrosis. IgG2 response to Pseudomonas aeruginosa lipopolysaccharide. Am. Rev. Respir. Dis. 133:418422.

41. Fick, R. B., Jr., G. P. Naegel, R. A. Matthay, and H. Y. Reynolds. 1981. Cystic fibrosis Pseudomonas opsonin. Inhibitory nature in an in vitro phagocytic assay. J. Clin. Invest. 68:899-914.

42. Hornick, D. B., and R. B. Fick, Jr. 1990. The immunoglobulin G subclass composition of immune complexes in cystic fibrosis. Implications for the pathogenesis of the Pseudomonas lung lesion. J. Clin. Invest. 86:1285-1292. 